Bundesgesundheitsbl 2013 $\cdot 56: 1349-1351$ DOI 10.1007/s00103-013-1829-8

Online publiziert: 25. September 2013

c) Springer-Verlag Berlin Heidelberg 2013

\title{
R. Seitz
}

Abt. Hämatologie/Transfusionsmedizin, Paul-Ehrlich-Institut, Langen

\section{Haben wir eine gute Blutversorgung?}

re Hepatitis-C-Virus, häufig zu den Spätfolgen Leberzirrhose und schließlich primärem Leberkarzinom führt. Diese Erfahrungen und Erkenntnisse lösten eine eindrucksvolle Weiterentwicklung in Wissenschaft und Technik aus. Die heute zur Spendertestung eingesetzten Testsysteme können Virusinfektionen in einem frühen Stadium entdecken. Bei der Herstellung von Plasmaderivaten können Viren inaktiviert oder eliminiert werden. Es gab aber auch sehr einschneidende Konsequenzen hinsichtlich der behördlichen Kontrolle und der in den Blutspendediensten einzuhaltenden Standards. So werden z. B. in Richtlinien, die vom Wissenschaftlichen Beirat der Bundesärztekammer im Einvernehmen mit dem Paul-Ehrlich-Institut aufgestellt und im Bundesanzeiger veröffentlicht werden, Kriterien zur Spenderauswahl und technische Anforderungen festgelegt.

Eine wesentliche Reaktion des Gesetzgebers auf die oben angesprochene Krise war das 1998 in Kraft getretene Transfusionsgesetz (TFG). Ein zentrales Anliegen dieses Gesetzes war die verbindliche Festschreibung von essenziellen Anforderungen zur Minimierung des Infektionsrisikos, z. B. die Spendertestung oder die Rückverfolgbarkeit über die gesamte Kette von der Blutspende bis zu den mit den gewonnenen Arzneimitteln behandelten Patienten. Dies ist aber nur ein Teil des Gesetzeszweckes, wie in $\$ 1$ TFG formuliert: „Zweck dieses Gesetzes ist es, nach Maßgabe der nachfolgenden Vorschriften zur Gewinnung von Blut und Blutbestandteilen von Menschen und zur Anwendung von Blutprodukten für eine sichere Gewinnung von Blut und Blutbestandteilen und für eine gesicherte und sichere Versorgung der Bevölkerung mit Blutprodukten zu sorgen und deshalb die Selbstversorgung mit Blut und Plasma auf der Basis der freiwilligen und unentgeltlichen Blutspende zu fördern.“

Es geht also nicht nur um die Qualität und Sicherheit der therapeutischen Blutprodukte, sondern auch um die gesicherte, d. h. quantitativ ausreichende und nachhaltig sichergestellte Versorgung der Patienten mit diesen unverzichtbaren Arzneimitteln. Um hier ggf. mit gesundheitspolitischen Maßnahmen eingreifen zu können, ist eine regelmäßige Erfassung der Versorgungssituation erforderlich. Hierfür wurden die Voraussetzungen im $₫ 21$ TFG geschaffen. Danach haben die Träger der Spendeeinrichtungen, die pharmazeutischen Unternehmer und die Einrichtungen der Krankenversorgung jährlich die Zahlen zu dem Umfang der Gewinnung von Blut und Blutbestandteilen, der Herstellung, des Imports und Exports und des Verbrauchs von Blutprodukten und Plasmaproteinen sowie die Anzahl der behandlungsbedürftigen Personen mit angeborenen Hämostasestörungen der zuständigen Bundesoberbehörde, d. h. dem Paul-Ehrlich-Institut, zu melden. Diese Meldeverpflichtung wurde mit Verkündigung des TFG im Jahr 1998 ohne Übergangsfrist in Kraft gesetzt, es mussten jedoch von allen Beteiligten zunächst die nötigen Voraussetzungen geschaffen werden. Ein großes Problem hierbei war die Heterogenität der zur Meldung verpflichteten Einrichtungen. In den Blutspendediensten war die erforderliche Dokumentation im Prinzip vorhanden, sodass die Zusammenfassung der Herstellungszahlen für die Meldungen zwar mit zusätzli- 
chem Aufwand verbunden, aber leistbar war. In der plasmaverarbeitenden Industrie sind prinzipiell die Herstellungszahlen verfügbar, es ist aber bei der globalen Operation der Hersteller oft schwierig, aus den Zahlen der Produktion an verschiedenen Standorten, Importen und Exporten, die in Deutschland verfügbaren Mengen darzustellen. Eine besondere Anforderung stellt die Meldepflicht für die Einrichtungen der Krankenversorgung dar, eine sehr inhomogene Gruppe, die von großen Kliniken der Maximalversorgung bis hin zu ambulanten Einrichtungen wie hämatologischen oder Dialysepraxen reicht. In all diesen Einrichtungen musste eine Dokumentation über den Verbrauch von Blutprodukten etabliert und die Zusammenführung der zu meldenden Zahlen organisiert werden.

Eine erhebliche Herausforderung bedeutete aber auch der Aufbau der erforderlichen Strukturen im Paul-Ehrlich-Institut, das bis dahin keine Aufgaben im Bereich von Datenerfassung und Statistik wahrgenommen hatte. Hier gelang es mit großem Engagement der Mitarbeiter, ein Konzept und eine Datenbankstruktur für die Erfassung der Daten zu entwickeln. Anfangs wurden entsprechende Formulare an die bekannten Meldepflichtigen verschickt, die von diesen ausgefüllt zurückgesandt werden mussten. Die Daten mussten dann im Paul-Ehrlich-Institut per Hand in die Datenbank eingegeben werden. Es zeigte sich, dass in der Anfangszeit die Daten zum Teil inkonsistent und insgesamt unvollständig waren. Dies lag teils an Schwierigkeiten der Dokumentation, Erfassung und Übertragung der Daten in die Meldebögen in den Einrichtungen. Das Ausbleiben von Meldungen aus zahlreichen Einrichtungen lag aber auch daran, dass das TFG zwar eine Meldepflicht, aber keine Sanktionen bei Nichtbefolgung vorsieht.

Um die Nutzerfreundlichkeit und -akzeptanz zu erhöhen und zu möglichst belastbaren Daten zu kommen, wurde eine externe Softwarefirma mit der Entwicklung einer webbasierten Datenbank-Anwendung beauftragt; seit 2007 ist die Online-Meldung durch die Einrichtungen selbst obligat. Die Erfassung der verfügbaren Mengen an Blutprodukten konnte nach der oben beschriebenen Anlauf- phase seit dem Jahr 2000 verbessert und stabilisiert werden. Inzwischen sind auch die Meldungen des Verbrauchs von Blutprodukten nicht zuletzt dank der umfassenden Aufklärung durch die Landesärztekammern und das Paul-Ehrlich-Institut von guter Qualität. Zusätzlich zu den Meldungen aus den Einrichtungen der Krankenversorgung werden Angaben wie Verkaufszahlen der Industrie und Apotheken genutzt.

Besondere Anstrengungen wurden unternommen, um solide Daten zu den im Gesetz angesprochenen Personen mit angeborenen Hämostasestörungen zu erhalten. Hier geht es z. B. um die Hämophilie, die meist eine lebenslange und sehr kostenintensive Behandlung erfordert. Schon die Ermittlung der Anzahl der behandlungsbedürftigen Personen mit angeborenen Hämostasestörungen ist durchaus nicht trivial, da weder diese Zahlen, noch die an der Hämophiliebehandlung beteiligten - also meldepflichtigen - Ärzte bis dahin zentral erfasst wurden. Es gab allerdings bereits seit den krisenhaften HIV-Übertragungen bei der Behandlung von Hämophiliepatienten auf Initiative von Prof. Wolfgang Schramm, LMU München, eine kontinuierliche Erfassung der Todesursachen dieser Patientengruppe. Auf seinen Vorschlag hin wurde als Kooperationsprojekt von Paul-Ehrlich-Institut, wissenschaftlicher Fachgesellschaft GTH (Gesellschaft für Thrombose- und Hämostaseforschung e.V.) und den beiden Patientenorganisationen DHG (Deutsche Hämophiliegesellschaft zur Bekämpfung von Blutungskrankheiten e.V.) und IGH (Interessengemeinschaft Hämophiler e.V.) das Deutsche Hämophilieregister (DHR) mit Sitz am Paul-Ehrlich-Institut ins Leben gerufen. Das mit einer Anschubfinanzierung vom Bundesministerium für Gesundheit geförderte DHR sammelt in einer Online-Datenbank detaillierte Behandlungsdaten mit der Einwilligung der Patienten und unter strenger Einhaltung der Datenschutzvorschriften. Die behandelnden Ärzte haben aber auch weiterhin, z. B. wenn Patienten nicht in die Eingabe ihrer individuellen Daten einwilligen, die Möglichkeit, die nach $\$ 21$ TFG geforderten kumulierten Behandlungsdaten verschiedener Patientengruppen zu melden.
Das DHR soll so der vollständigen Erfassung der Anzahl der behandlungsbedürftigen Patienten und des Bedarfs an Gerinnungspräparaten dienen. Darüber hinaus soll sich das DHR zu einem Instrument der Qualitätssicherung in der Hämophiliebehandlung und der klinischen Forschung zur Therapieoptimierung entwickeln. Dieses Ziel kann allerdings erst in einigen Jahren nach Einbringung von Längsschnittdaten durch die Hämophiliebehandler angestrebt werden. Patientenregister dürften, insbesondere bei seltenen Erkrankungen wie der Hämophilie, zunehmende Bedeutung erlangen und gerade im internationalen Vergleich wertvolle Erkenntnisse liefern. Daher wurde von Anfang an ein Abgleich mit den von Registern anderer Länder erfassten Parametern angestrebt.

Nach dem Auftrag im TFG stellt die zuständige Bundesoberbehörde (PaulEhrlich-Institut) die gemeldeten Daten anonymisiert in einem Bericht zusammen und macht diesen bekannt. Sie hat melderbezogene Daten streng vertraulich zu behandeln. Während in den ersten Berichten vor allem die Darlegung der Methodik und die Überwindung der Anlaufschwierigkeiten thematisiert werden mussten, gelang es ab 2000, zunehmend belastbare Daten zu gewinnen, die auch die Darstellung von Trends in der Versorgung erlauben. Dabei zeigt sich über die Jahre, wie erneut in dem aktuellen Bericht (Henseler et al., Bericht zur Meldung nach $₫ 21$ TFG für die Jahre 2010 bis 2012) in dieser Ausgabe belegt, dass das Aufkommen an Blutspenden in Deutschland im Europäischen Vergleich in der Spitzengruppe liegt.

Das schließt nicht aus, dass gerade in der Ferienzeit hin und wieder Engpässe auftreten, die auch zu einer Verschiebung planbarer operativer Eingriffe führen können. Zudem wird es angesichts der demografischen Entwicklung mit tendenziell weniger jungen Blutspendern und mehr behandlungsbedürftigen Älteren erforderlich sein, die Motivationskampagnen für die Blutspende nicht nur weiterzuführen, sondern zu intensivieren. Entsprechende Medienberichte werden immer wieder aufgegriffen von Personen oder Gruppen, die aus medizinischen Gründen nicht zur Blutspende zu- 
gelassen werden. Es wird geklagt, dass die Spenderauswahlkriterien zu streng seien und so dringend benötigte Blutspenden verhindert würden. Der Wunsch, mit Blutspenden Menschen zu helfen, verdient große Anerkennung, und es ist verständlich, wenn eine Verweigerung dieser Möglichkeit als Zurückweisung empfunden wird. Angesichts der potenziellen Risiken hat jedoch die Sicherheit der mit Blutprodukten behandelten Patienten absolute Priorität. Der Bericht in dieser Ausgabe (Bericht zur Meldung nach $\$ 21$ TFG für die Jahre 2010 bis 2012) zeigt eindrucksvoll, dass wir in Deutschland nicht nur sehr sichere Blutprodukte, sondern auch eine im internationalen Vergleich gute und nachhaltig sichergestellte Versorgung der Patienten mit diesen unverzichtbaren Arzneimitteln haben.

Ihr

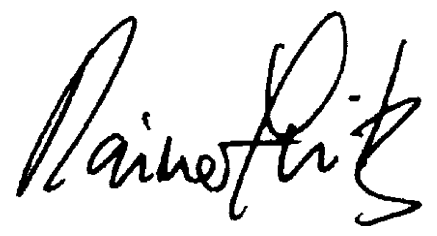

Rainer Seitz

\section{Korrespondenzadresse}

\begin{tabular}{|l|l} 
& $\begin{array}{l}\text { Prof. Dr. R. Seitz } \\
\text { Abt. Hämatologie/ } \\
\text { Transfusionsmedizin, } \\
\text { Paul-Ehrlich-Institut } \\
\text { Paul-Ehrlich-Str.51-59, } \\
\text { 63225 Langen } \\
\text { Rainer.Seitz@pei.de }\end{array}$ \\
\hline
\end{tabular}

